\title{
Dynamics of morphological changes for mitochondrial fission and fusion ${ }^{\dagger}$
}

\author{
WANG ShiQi ${ }^{1,2,4}$, FU ChangLiang ${ }^{1,2}$, ZHANG Yan $^{1,2}$, CHEN Quan ${ }^{3} \&$ LONG Mian $^{1,2^{*}}$ \\ ${ }^{1}$ Key Laboratory of Microgravity (National Microgravity Laboratory), Beijing 100190, China; \\ ${ }^{2}$ Center for Biomechanics and Bioengineering, Institute of Mechanics, Chinese Academy of Sciences, Beijing 100190, China; \\ ${ }^{3}$ State Key Laboratory of Biomembrane and Membrane Biotechnology, Institute of Zoology, Chinese Academy of Sciences, \\ Beijing 100101, China; \\ ${ }^{4}$ Institute of Vertebrate Paleontology and Paleoanthropology, Chinese Academy of Sciences, Beijing 100044, China
}

Received September 2, 2009; accepted September 28, 2009; published online March 25, 2010

\begin{abstract}
Mitochondria experience continuous fusion and fission in a living cell, but their dynamics remains poorly quantified. Here a theoretical model was developed, upon a simplified population balance equation (PBE), to predict the morphological changes induced by mitochondrial fission and fusion. Assuming that both fission and fusion events are statistically independent, the survival probability of mitochondria staying in the fission or fusion state was formulated as an exponentially-decayed function with time, which depended on the time-dependent distribution of the mitochondrial volume and the fission and fusion rates. Parametric analysis was done for two typical volume distributions. One was Gamma distribution and the other was Gaussian distribution, derived from the measurements of volume distribution for individual mitochondria in a living cell and purified mitochondria in vitro. The predictions indicated that the survival probability strongly depended on morphological changes of individual mitochondria and was inversely correlated to the fission and fusion rates. This work provided a new insight into quantifying the mitochondrial dynamics via monitoring the evolution of the mitochondrial volume.
\end{abstract}

mitochondria, morphology, fission, fusion, dynamics, modeling

PACS: 87.16.-b, 87.16.Ac, 87.16.Tb, 87.16.Uv

Mitochondrial functionality is crucial to cell life and death. In a live cell, mitochondria tend to form a highly interconnected network and undergo continuous fission and fusion $[1,2]$. Such complex network is advantageous, as compared to the isolated individual mitochondria, in cellular metabolism, mitosis, and apoptosis as well as in organelle biogenesis and energy conservation. For example, mitochondrial fission regulates the cellular biological activity levels at different sites [3], while the fused mitochondria coordinate mass transport, energy transfer, and signal transduction, which transmits membrane potential $\Delta \Psi_{\mathrm{m}}[4,5]$. Moreover, the isolated mitochondria can be fused in vitro to form the

*Corresponding author (email: mlong@imech.ac.cn)

$\dagger$ Contributed by LONG Mian large-sized organelles [6]. Thus, the balance of mitochondrial fission and fusion is a prerequisite for regulating the biological activities in a live cell.

It has long been noticed that the morphology of individual mitochondria is correlated with cell cycle, as observed in a highly-tubular network at interphase but a highlyfragmented structure in mitosis [7]. One interpretation is that mitochondrial fission makes it easier to allocate them into daughter cells, but it is hard to understand why the fraction of tubular network is increased at telephase [7]. The knock-out of mitochondrial proteins also induces the dramatic morphological change of individual mitochondria [8], but the underlying mechanisms for how mitochondrial morphology varies with time are still unclear. Thus, it is required to quantify the dynamics of morphological changes 
induced by mitochondrial fission and fusion.

A few studies have attempted to quantify, respectively, the mitochondrial morphology and fission and fusion dynamics in a live cell at sub-cellular level. On the one hand, for example, the spatial distribution of mitochondria in axons of living neurons was found to follow a Poisson distribution [9,10]. Morphological changes of mitochondrial network were quantified using a Fourier imaging correlation spectroscopy assay [11]. The fractal property of moving trajectory of sub-cellular vesicles [12] and three-dimensional (3D) movement of cytoplasmic membrane vesicles [13] were also investigated. These works, however, are quite preliminary in quantifying the dynamics of mitochondrial morphology using the simplified models. On the other hand, several analyses of mitochondrial dynamics were also done in mitotic or budding yeast as well as in yeast in meiosis [14-16]. For example, the antetograde and retrograde velocities of mitochondrial movement were balanced and no net movement was found in budding yeast [14]. Mitochondrial fission and fusion rates yielded the same values in mitotic yeast [15]. The fraction of fragmented mitochondria was enhanced in yeast sporulation [16]. Although these pioneering works provided some quantitative evidence for mitochondrial movement and dynamics, it is still far away from quantifying the mitochondrial fission and fusion at sub-cellular level with methodological difficulties [17]. Therefore, not only the 3D mitochondrial network needs to be visualized experimentally since it is inter-connected in 3D configuration and evolutes in a time-dependent pattern, but the theoretical prediction is also required to correlate the morphological changes of individual mitochondria with their fission and fusion dynamics.

Here we developed a theoretical model, upon a modified population balance equation (PBE) [18], to describe the evolution of mitochondrial morphology mediated by their fission and fusion activities. Experimental observations of volume distribution of individual mitochondria in a living cell or of purified mitochondria in vitro were employed to predict the time dependence of survival probability of a mitochondrion in fission or fusion state. Our modeling provided a new insight into the dynamics of mitochondrial fission and fusion from the viewpoint of morphological changes.

\section{Experimental procedures}

Cells and reagents-Mitotrack-CMX-ROS was purchased from Cell Signaling Technology (Boston, USA). All the other reagents were from Beijing Chemical Reagents Company (Beijing, China) with the highest purity.

Human epithelial carcinoma (Hela) cells obtained from ATCC were grown up in Dulbecco's modified Eagle medium (DMEM) (Hyclone, Logan, USA) supplemented with
$10 \%$ heat-inactivated fetal bovine serum (Gibco, Tulsa, USA) and $1 \%$ of penicillin and streptomycin each (Hyclone, Logan, USA). Harvested cells were placed on a culture plate with a transparent window, and treated with $1 \mu \mathrm{M}$ Mitotrack-CMX-ROS for $20 \mathrm{~min}$ before laser confocal microscopic observation.

Visualization of mitochondrial morphology-A twophoton laser scanning microscopy (Carl Zeiss LSM 510 Meta, Germany) was used to monitor the individual mitochondria in a living cell. A culture plate was placed onto the stage of inverted microscope and the time-lapsed images were recorded using a $60 \times$ oil immersed lens (Carl Zeiss Z500, Germany). A single cell was sliced at $1 \mu \mathrm{m}$ interval along $z$-direction with a spatial resolution of $0.05-0.14$ $\mu \mathrm{m} /$ pixel, and the scanning time per slice varied in $0.1-1.3 \mathrm{~s}$ in a temporal resolution of $0.80-1.68 \mu \mathrm{s} /$ pixel. One cycle of cell slicing (including the scanning time for a specific slice and switch duration between two sequential slices) was pre-set at $12-15 \mathrm{~s}$ per cycle. Cell slicing was performed consecutively from one to another slice until it was completed from the bottom to the top.

Three-dimensional (3D) reconstruction-A course 3D reconstruction approach was developed to visualize the time-lapsed evolution of mitochondrial volume in the $x-y$ plane and along the $z$-direction. Each slice was digitalized as a black and white image and the contour of an isolated mitochondrion was determined. For filtering out the background noise, a threshold of grey value was set for each cell (Figures 1(a) and (c)). Individual mitochondria in arbitrary three sequential slices were respectively colored in red, green, and blue from the bottom to the top, and then aligned together. This procedure was repeated from the bottom $(z=$ 0 ) to the top ( $z=H$, where $H$ is the maximum thickness of a cell) (Figures 1(b) and (d)). The volume of an isolated mitochondrion was calculated as the product of mean projected area and its height across all slices by simplifying it as a cylinder with a uniform diameter. The volumes for all the mitochondria in the cell were estimated in each cycle and all the values obtained from the total 31-33 cycles were then pooled together for further analyses.

\section{Theoretical models}

\subsection{Basic equations}

In the current work, the population balance equation (PBE) was used as the theoretical framework to quantify the dynamics of mitochondrial fission and fusion. The starting point of our modeling was to describe the evolution of number density, $n(V, t)$, of the organelles [18],

$$
\frac{\partial n(V, t)}{\partial t}=\int_{V_{0}}^{V / 2} k_{a}(w, V-w) n(w, t) n(V-w, t) \mathrm{d} w
$$




$$
\begin{aligned}
& -\int_{V_{0}}^{+\infty} k_{a}(w, V) n(w, t) n(V, t) \mathrm{d} w \\
& +\int_{V}^{+\infty} k_{d}(w) n(w, t) \gamma(w) \rho_{s}(w, V) \mathrm{d} w \\
& -k_{d}(V) n(V, t),
\end{aligned}
$$

where $V$ is the volume of organelle $\left(V_{0} \leqslant V \leqslant+\infty\right)$ at time $t$ $(0 \leqslant t \leqslant+\infty)$ (in $\left.\mu \mathrm{m}^{3}\right), V_{0}$ defines the minimum threshold of $V$ for available fission (as seen below) (in $\mu \mathrm{m}^{3}$ ), $\gamma(w)$ is the number of organelles with an arbitrary volume of $w, \rho_{\mathrm{s}}(w$, $V)$ is the volume distribution of $\gamma(w)$, and $k_{d}(V)$ and $k_{a}(w, V)$ are the fission and fusion rates per volume, respectively (in $\mathrm{s}^{-1} \mu \mathrm{m}^{-3}$ ). The first term on the right-hand side describes the fusion rate of two organelles whose volumes add up to $V$. The second term represents the fission rate of organelles with volume $V$ by their collision and binding with an organelle with any volume. The third term accounts for the production of organelles with volume $V$ by break-up of organelles with volume $w(V \leqslant w<+\infty)$. The last term denotes the disappearance of organelles with volume $V$ due to their break-up into organelles with volume $w(0 \leqslant w \leqslant V)$.

Our modeling on mitochondrial fission and fusion was based on three key assumptions: 1) A single organelle is simplified as a cylinder-like rod with a uniform diameter and the fission or fusion takes place at an arbitrary element, $\mathrm{d} w$, with the same probability (Figures 1(e) and (f)); 2) There exists a minimum threshold of $V$, i.e. $V_{0}$, below which the organelle is still able to bind with an organelle with any volume but unable to break up into two smaller organelles (Figure 1(e)); 3) Only a single collision between two organelles is taken into account at any time.

Since the analytical solution to eq. (1) is not available, numerical simulations are required to calculate the fission and fusion rates. Briefly, eq. (1) can be discretized using the distribution of volume, $V\left(t_{i}\right)$, obtained from measurements at a pre-set time series $\left\{t_{1}, t_{2}, \ldots, t_{i}, \ldots, t_{N}\right\}$ (where $t_{N}$ is the end moment of observation), and then solved by a finite differentiation method to estimate the time courses of $k_{d}(V)$ and $k_{a}(w, V)$ [18]. Since the iteration is quite labor- and time-consuming, an alternative theoretical approach was proposed in the current work.

\subsection{Simplified models}

The simplification for mitochondrial dynamics is to decouple the fission and fusion processes by assuming that the fission or fusion event is stochastically independent of each other, that is, the probability of simultaneous occurrence of fission and fusion is quite low for an isolated organelle. This seemed to be reasonable from the observation that only
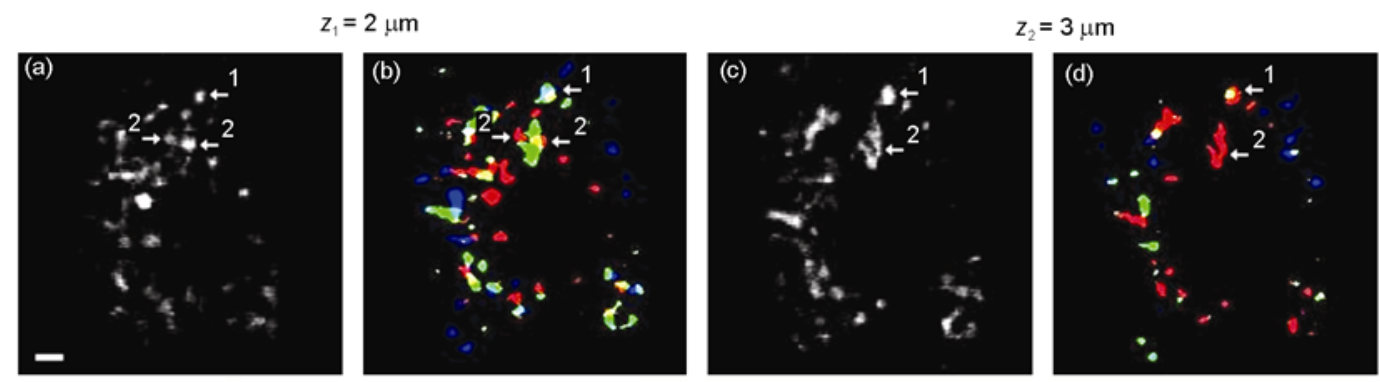

(e)
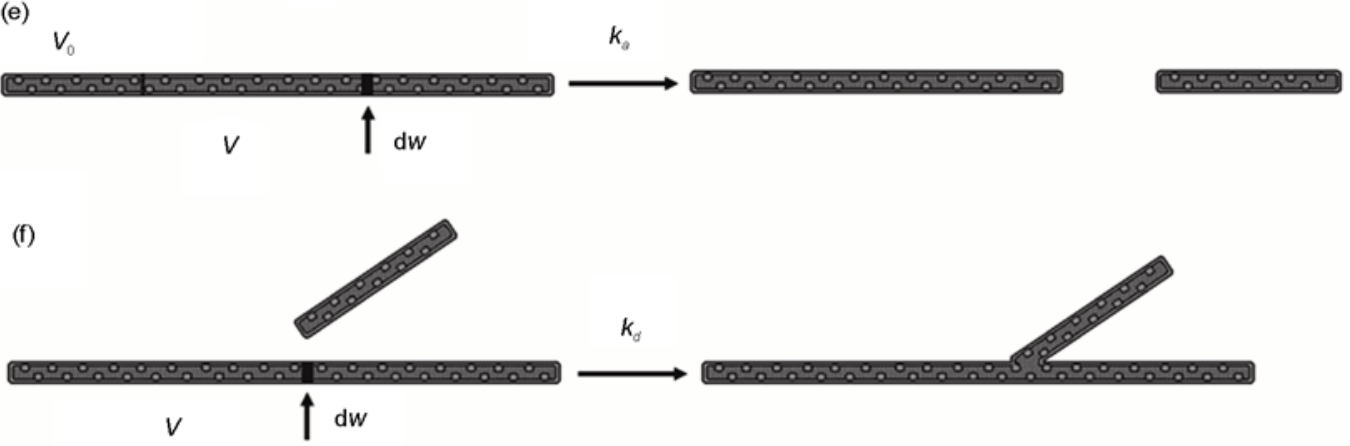

Figure 1 (a)-(d) Volume estimations of individual mitochondria in a mitotic Hela cell at two focus planes of $z_{1}=2 \mu \mathrm{m}((\mathrm{a}),(\mathrm{b}))$ and $z_{2}=3 \mu \mathrm{m}((\mathrm{c}),(\mathrm{d}))$ from the bottom $(\operatorname{Bar}=2 \mu \mathrm{m})$. The white and black images were originally obtained from a dual-photon laser scanning microscopy $((\mathrm{a})$, (c)). The colored images were the 3D reconstructed ones where one set of three sequential images at $z_{j}, z_{j+1}$, and $z_{j+2}$ were colored by red, green, and blue, respectively, and then superposed into a single image ((b),(d)). This process was repeated using another set of three sequential images at $z_{j+1}, z_{j+2}$, and $z_{j+3}$, until all the images were reconstructed from the bottom to the top of the cell within the entire duration. Arrows and numbers indicate two typical mitochondria. Note that the \#2 mitochondrion, primarily observed as "two" individuals in the $z_{1}=2 \mu \mathrm{m}$ panel, was finally confirmed as a "single" mitochondrion using the 3D reconstructed images ((b), (d)). (e), (f) Schematic of theoretical modeling for mitochondrial fission from (e) and fusion to (f) a volume element, dw. Here $V$ is the mitochondrial volume and $V_{0}$ is the minimum threshold for fission. $k_{d}$ and $k_{a}$ are the fission and fusion rates, respectively. 
$13.4 \%(15.2 \%, 8.1 \%$, and $17.0 \%$ from three individual cells) of mitochondria was found to present the simultaneous fission and fusion. In a typical measurement, one can monitor the evolution of the number and size (or volume) and the occurrence of fission or fusion events for individual mitochondria. It was also noticed that the organelle exhibited a dynamic transition between fission and fusion with varied volumes $[7,8,19]$. Here the survival probability of the organelle with volume $V$ not to break up at time $t, P_{d V}$, follows:

$$
\frac{\mathrm{d} P_{d V}}{\mathrm{~d} t}=-\left[\left(V-V_{0}\right) \times k_{d}\right] P_{d V}
$$

If we set $P_{d V}=1$ at $t=0$ and $V_{0}$ is a constant, the solution to eq. (2) yields at a fixed $V$ :

$$
P_{d V}=\exp \left[-\left(V-V_{0}\right) \times k_{d} \times t\right]
$$

Similarly, the survival probability of an organelle with volume $V$ not to fuse at time $t, P_{a V}$, gives:

$$
\frac{\mathrm{d} P_{a V}}{\mathrm{~d} t}=-\left(V \times k_{a}\right) P_{a V} .
$$

Again, with $P_{a V}=1$ at $t=0$, the solution to eq. (4) satisfies at a fixed $V$ :

$$
P_{a V}=\exp \left(-V \times k_{a} \times t\right)
$$

Note that an implicit assumption that the fission or fusion rate $\left(k_{d}\right.$ or $\left.k_{a}\right)$ does not vary with time $t$ was used when integrating eqs. (2) and (4) with time $t$, since the fission or fusion occurs at a commensurate volume element, $\mathrm{d} w$ with same opportunity (Figures 1(e) and (f)). Moreover, there exists a distribution of mitochondrial volume at a given time $t$, that is, a probability density function $\rho(V)$. Thus, the time dependence of mean survival probability yields:

$$
\overline{P_{d V}}=\int_{V_{0}}^{+\infty} P_{d V} \rho(V) \mathrm{d} V
$$

or

$$
\overline{P_{a V}}=\int_{0}^{+\infty} P_{a V} \rho(V) \mathrm{d} V
$$

Two volume distributions were used in the current work. One is the Gamma distribution with a probability density function of $V$ :

$$
\rho(V)=\frac{\beta^{\alpha}}{\Gamma(\alpha)}\left(V-V_{0}\right)^{\alpha-1} \mathrm{e}^{-\beta\left(V-V_{0}\right)} \quad \text { (for fission) }
$$

or

$$
\rho(V)=\frac{\beta^{\alpha}}{\Gamma(\alpha)} V^{\alpha-1} \mathrm{e}^{-\beta V} \quad \text { (for fusion), }
$$

here $\Gamma(\alpha)$ is the Gamma function and $\alpha$ and $\beta$ are the adjustable parameters. For the sake of fitting the measured volume distribution, the accumulative probability function is also given:

$$
F(V)=\frac{\Gamma\left(\alpha, \beta\left(V-V_{0}\right)\right)}{\Gamma(\alpha)} \text { (for fission) }
$$

or

$$
F(V)=\frac{\Gamma(\alpha, \beta V)}{\Gamma(\alpha)} \text { (for fusion), }
$$

here $\Gamma(\alpha, z)=\int_{0}^{z} t^{\alpha-1} \mathrm{e}^{-t} \mathrm{~d} t$ is incomplete Gamma function.

The other possible volume distribution is the Gaussian distribution, as observed in the size distribution for individual mitochondria purified from cells and re-suspended in vitro in buffer solution [20], with the probability density function,

$$
\rho(V)=\frac{1}{\sigma \sqrt{2 \pi}} \mathrm{e}^{-\left(V-V_{0}-\mu\right)^{2} /\left(2 \sigma^{2}\right)} \quad \text { (for fission) }
$$

or

$$
\rho(V)=\frac{1}{\sigma \sqrt{2 \pi}} \mathrm{e}^{-(V-\mu)^{2} /\left(2 \sigma^{2}\right)} \quad \text { (for fusion), }
$$

and the accumulative probability function,

$$
F(V)=\frac{1}{2}\left(1+\operatorname{Erf}\left(\frac{V-V_{0}-\mu}{\sqrt{2} \sigma}\right)\right) \text { (for fission) }
$$

or

$$
F(V)=\frac{1}{2}\left(1+\operatorname{Erf}\left(\frac{V-\mu}{\sqrt{2} \sigma}\right)\right) \text { (for fusion) }
$$

here $\operatorname{Erf}(z)=\frac{2}{\sqrt{\pi}} \int_{0}^{z} \exp \left(-t^{2}\right) \mathrm{d} t$ is the Gaussian error function, $\mu$ is the mean volume, and $\sigma$ is the standard deviation of the mean.

Eqs. (6a) and (6b) contain analytical solutions when $V$ is Gamma-distributed. Substituting eqs. (3) and (7a) into eq. (6a) or eqs. (5) and (7b) into eq. (6b) for the integral, we get

$$
\overline{P_{d V}}=\left(\frac{\beta}{k_{d} t+\beta}\right)^{\alpha},
$$

or

$$
\overline{P_{a V}}=\left(\frac{\beta}{k_{a} t+\beta}\right)^{\alpha}
$$


Note that shifting the lower limit of the integral in eq. (6a) from $V_{0}$ to 0 results in the same expression as the integral in eq. (6b) (except the rates $k_{d}$ and $k_{a}$ ) (eqs. (10a) and (10b)).

Again, substituting eqs. (3) and (9a) into eq. (6a) or eqs. (5) and (9b) into eq. (6b) for the integral yields for Gaussian-distributed volume:

$$
\begin{aligned}
\overline{P_{d V}}= & \frac{1}{2} \exp \left(\frac{k_{d} t\left(-2 \mu+k_{d} t \sigma^{2}\right)}{2}\right) \\
& \times\left(1+\operatorname{Erf}\left(\frac{\mu-k_{d} t \sigma^{2}}{\sqrt{2} \sigma}\right)\right)
\end{aligned}
$$

or

$$
\begin{aligned}
\overline{P_{a V}}= & \frac{1}{2} \exp \left(\frac{k_{a} t\left(-2 \mu+k_{a} t \sigma^{2}\right)}{2}\right) \\
& \times\left(1+\operatorname{Erf}\left(\frac{\mu-k_{a} t \sigma^{2}}{\sqrt{2} \sigma}\right)\right) .
\end{aligned}
$$

Note that the integral eq. (6a) or (6b) is in the regime of $(0$, $+\infty)$. Therefore, eqs. (12a) and (12b), which did not reach unity at $t=0$, were re-normalized to 1.0 at $t=0$.

$$
\overline{P_{d V}}=\frac{\exp \left(\frac{k_{d} t\left(-2 \mu+k_{d} t \sigma^{2}\right)}{2}\right) \times\left(1+\operatorname{Erf}\left(\frac{\mu-k_{d} t \sigma^{2}}{\sqrt{2} \sigma}\right)\right)}{\left(1+\operatorname{Erf}\left(\frac{\mu}{\sqrt{2} \sigma}\right)\right)}
$$

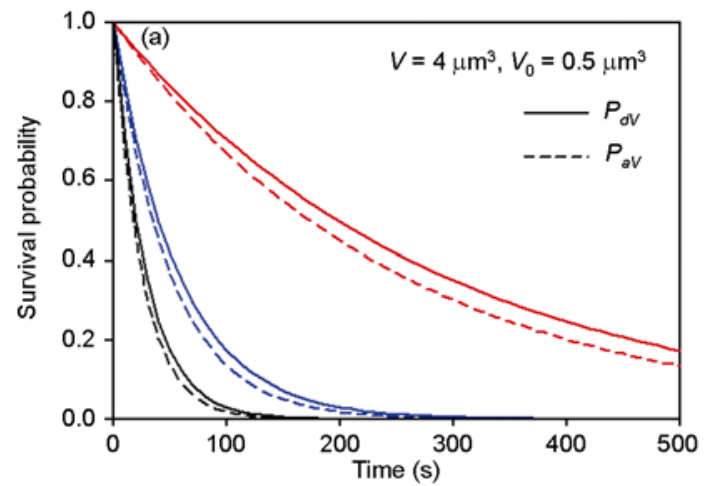

or

$$
\overline{P_{a V}}=\frac{\exp \left(\frac{k_{a} t\left(-2 \mu+k_{a} t \sigma^{2}\right)}{2}\right) \times\left(1+\operatorname{Erf}\left(\frac{\mu-k_{a} t \sigma^{2}}{\sqrt{2} \sigma}\right)\right)}{\left(1+\operatorname{Erf}\left(\frac{\mu}{\sqrt{2} \sigma}\right)\right)} .
$$

\section{Results and discussion}

\subsection{Determination of survival probabilities}

We firstly predicted the evolution of individual mitochondria experiencing continuous fission and fusion. At a set of parameters summarized in Table 1 , both $P_{d V}$ (solid lines) and $P_{a V}$ (dashed lines) decreased exponentially with time at a fixed volume $V=4 \mu \mathrm{m}^{3}$ (Figure 2(a)) or with volume at a fixed time $t=200 \mathrm{~s}$ (Figure 2(b)). It was also indicated that $P_{d V}$ or $P_{a V}$ shifted leftwards when $k_{d}$ or $k_{a}$ increased from $0.001,0.005$, to $0.01 \mathrm{~s}^{-1} \mu \mathrm{m}^{-3}$ (Table 1), indicating that the higher the activity of fission or fusion was, the lower the survival probability yielded. Note that the rightwards-shift of $P_{d V}$ (solid lines in Figure 2(b)), as compared to those for $P_{a V}$ (dashed lines in Figure 2(b)), was attributed to the minimum threshold $V_{0}$ for mitochondrial fission, as seen in eqs. (3) and (5).

\subsection{Varied volume distributions with time}

Different individual mitochondria have varied volumes at a

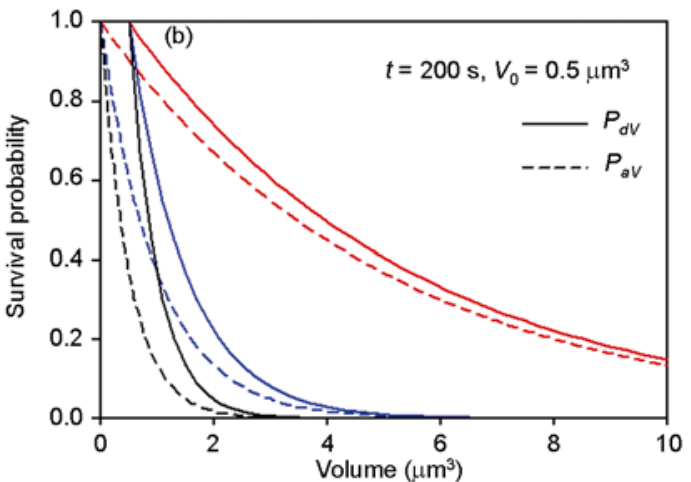

Figure 2 Time courses of survival probabilities $P_{d V}$ (solid lines) and $P_{a V}$ (dashed lines), calculated using eqs. (3) and (5), for mitochondrial fission and fusion at a fixed volume $V=4.0 \mu \mathrm{m}^{3}$ (a) or at a fixed time $t=200 \mathrm{~s}$ (b). Here the minimum threshold $V_{0}$ was set to be $0.5 \mu \mathrm{m}^{3}$, and $k_{d}$ and $k_{a}$ were set to be 0.001 (red lines), 0.005 (blue lines), and 0.010 (black lines) $\mathrm{s}^{-1} \mu \mathrm{m}^{-3}$, respectively.

Table 1 Parameters used in the predictions

\begin{tabular}{ccccc}
\hline Parameters & & \multicolumn{5}{c}{ Values } \\
\hline$k_{d}\left(\mathrm{~s}^{-1} \mu \mathrm{m}^{-3}\right)$ & 0.001 & 0.005 & 0.01 & \\
$k_{a}\left(\mathrm{~s}^{-1} \mu \mathrm{m}^{-3}\right)$ & 0.001 & 0.005 & 0.01 & \\
$(\alpha, \beta)\left(\bullet, \mu \mathrm{m}^{-3}\right)$ & $(0.5,1.0)$ & $(1.0,1.0)$ & $(1.5,1.0)$ & $(1.0,0.5)$ \\
$\mu \pm \sigma\left(\mu \mathrm{m}^{3}\right)$ & $0.5 \pm 0.5$ & $1.0 \pm 0.5$ & $1.5 \pm 0.5$ & $1.0 \pm 0.1$ \\
\hline
\end{tabular}


given time, which yields a volume distribution [7,19]. Here we measured the volumes for all the mitochondria in an intermitotic Hela cell and fitted the accumulative probability distribution using eq. (8b). It was found that the prediction (line) was in excellent agreement with the data (points) (Figure 3(a)), which returned the parameters of $\alpha=1.00$ and $\beta=0.68 \mu \mathrm{m}^{-3}\left(R^{2}=0.995\right)$. To monitor the variation of volume distribution with time, we also determined the time dependence of the parameters by fitting the data at different time points ( 31 data sets at the interval of $15 \mathrm{~s} ; R^{2}=0.986-$ 0.998). As exemplified in Figure 3(b), both the parameters of $\alpha$ (open circles) and $\beta$ (solid circles) retained the similar values at $t<220 \mathrm{~s}$, and then exhibited an ascending phase to reach the peaks at $t \sim 310 \mathrm{~s}$ followed by a descending phase up to $450 \mathrm{~s}$. The values were averaged over an entire period of observation, which turned to be $\bar{\alpha}=1.24 \pm 0.31$ (dashed line for the mean) and $\bar{\beta}=(0.93 \pm 0.38) \mu \mathrm{m}^{-3}$ (solid line for the mean) (Figure $3(\mathrm{~b})$ ). We also measured the goodness-of-fit for the volume distribution in a mitotic Hela cell (33 data sets at the interval of $12 \mathrm{~s} ; R^{2}=0.982-0.997$ ) and obtained the mean values of $\bar{\alpha}=(1.23 \pm 0.55)$ and $\bar{\beta}=$ $(1.70 \pm 1.35) \mu^{-3}$ from the time course of the fitted parameters (data not shown). These results indicated that the Gamma-distributed volume of individual mitochondria varied with time in a living cell and that the lower $\beta$ promoted the narrower distribution in an intermitotic Hela cell.

We further tested the volume distribution of individual mitochondria purified from living cells and re-suspended in buffer solution. This is still biologically-relevant since those purified mitochondria were able to complete, at least, the in vitro fusion and to form a large-sized organelle [6]. Here, we borrowed the data sets of size distribution of purified mitochondria from human embryonic T-Rex-293 kidney cells where the diameter was most likely Gaussian-distributed [20]. We re-calculated the volumes of purified mitochondria using $V=1 / 6 \pi D^{3}$ and obtained the accumulative probability distribution of volume (points in Figure 3(c)). The measured distribution fitted well with the prediction using eq. (10b) (line in Figure 3(c); $R^{2}=0.987$ ), which returned the parameters of $\mu=0.79 \mu \mathrm{m}^{3}$ and $\sigma=0.83 \mu \mathrm{m}^{3}$. Note that the time dependence of the parameters was no longer tested since the volume distribution did not vary with time in this regard.

\subsection{Parametric analysis of mean survival probability}

Next we tested the effect of mitochondrial fission and fusion rates on mean survival probabilities at a defined volume distribution. Note that both $\overline{P_{d V}}$ and $\overline{P_{a V}}$ have the same forms when $k_{d}=k_{a}$ whether the minimum threshold $V_{0}$ is introduced or not. Upon the above predictions, $(\alpha, \beta)$ were set to be $(1.0,1.0)\left(\bullet, \mu \mathrm{m}^{-3}\right)$ for Gamma-distribution and $(\mu, \sigma)$ to be $(1.0,0.5)\left(\mu \mathrm{m}^{3}\right)$ for Gaussian distribution.
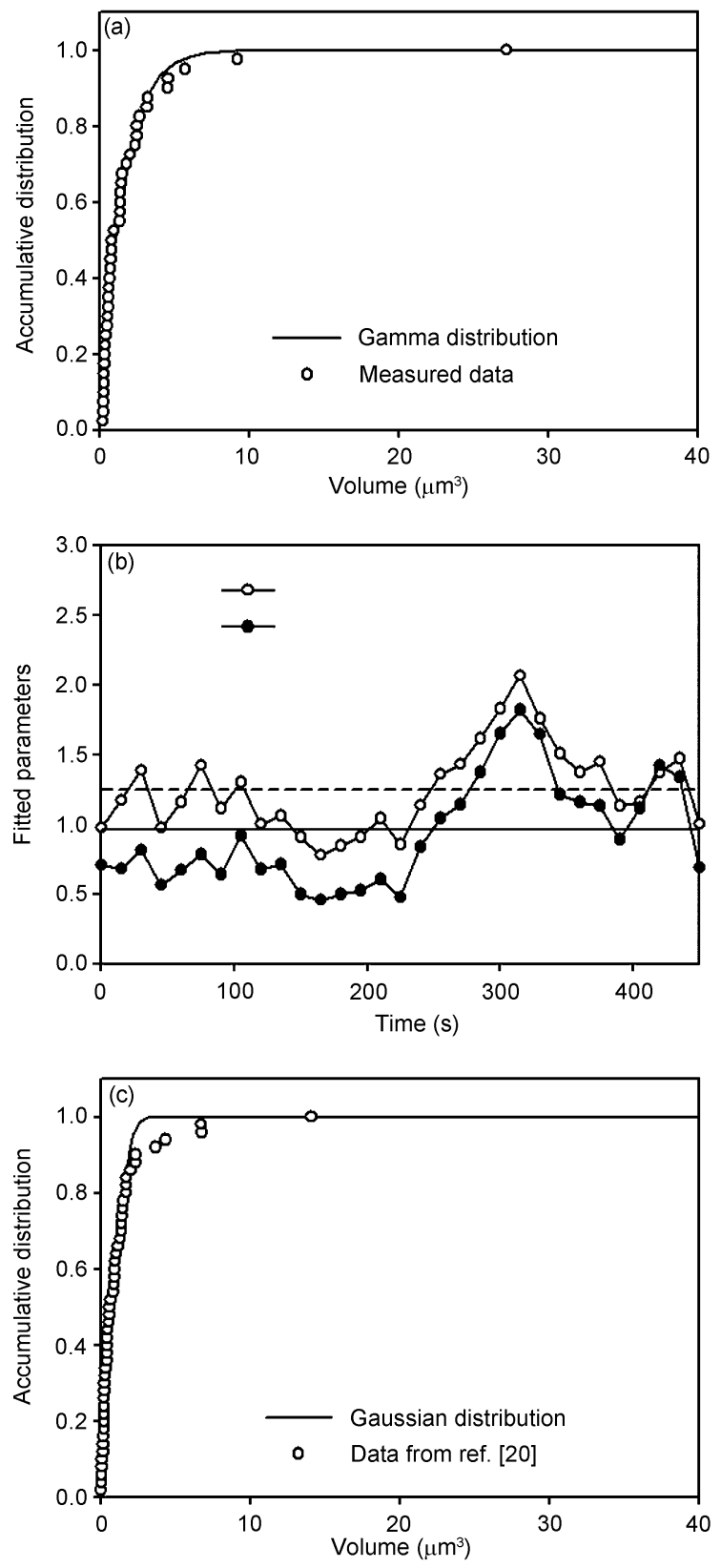

Figure 3 (a) Typical volume distribution of individual mitochondria at the interphase in a Hela cell. Data (points) was presented as the accumulative probability distribution of the mitochondrial volume before being compared with the prediction fitted using eq. (8b) (solid line). (b) Time dependence of parameters $\alpha$ (open circles) and $\beta$ (solid circles) for a Gamma distribution defined by eq. (7b). Dashed line and solid line are denoted as the mean values of $\alpha$ and $\beta$ with entire observation, respectively. (c) Typical volume distribution of purified mitochondria from human T-Rex-293 kidney cells. Data (points) adopted from our previous work [20] was presented as the accumulative probability distribution of the mitochondrial volume before being compared with the prediction fitted using eq. (10b) (solid line).

Time dependence of mean survival probability (same for $\overline{P_{d V}}$ and $\overline{P_{a V}}$ ) was determined via eqs. (11a), (11b) and (13a), (13b) at systematically-varied rates of $k_{d}=k_{a}=0.001$, 0.005 , and $0.01 \mathrm{~s}^{-1} \mu \mathrm{m}^{-3}$ (Table 1). It was found that $\overline{P_{d V}}$ 
or $\overline{P_{a V}}$ decreased exponentially with time and the curve shifted leftwards when $k_{d}$ or $k_{a}$ increased (Figures 4 (a) and (b)) whether the mitochondrial volume is Gamma- or Gaussian-distributed, which was the same as $P_{d V}$ or $P_{a V}$ found at a fixed volume (Figure 2(a)). The predictions also indicated that the higher the fission or fusion activity was, the lower the mean survival probability yielded.

We further tested the impact of adjustable parameters at the fixed rates of $k_{d}=k_{a}=0.005 \mathrm{~s}^{-1} \mu \mathrm{m}^{-3}$. Here the survival probability was compared between two sets of systematically-varied parameters of $\alpha=0.5,1.0,1.5$ at a given $\beta$ of $1.0 \mu \mathrm{m}^{-3}$ and $\beta=0.5,1.0,1.5 \mu \mathrm{m}^{-3}$ at a given $\alpha$ of 1.0 for Gamma-distributed volume (Table 1). The predictions demonstrated that $\overline{P_{d V}}$ or $\overline{P_{a V}}$ shifted leftwards when $\alpha$ increased from 0.5 (solid line), 1.0 (dashed line) to 1.5 (dotted line) (Figure 5(a)) but moved rightwards when $\beta$ increased from 0.5 (solid line), 1.0 (dashed line) to 1.5 (dotted line) $\mu \mathrm{m}^{-3}$ (Figure 5(b)), suggesting that high $\alpha$ value accelerated the decay of mean survival probability $\overline{P_{d V}}$ or $\overline{P_{a V}}$ whereas high $\beta$ value slowed down the decrease of $\overline{P_{d V}}$ or $\overline{P_{a V}}$ with time. Parametric analysis was also done for Gaussian - distributed volume where two sets of parameters of $\mu=0.5,1.0$, and $1.5 \mu \mathrm{m}^{3}$ at a fixed $\sigma=0.5$ $\mu \mathrm{m}^{3}$ and $\sigma=0.1,0.5$, and $1.0 \mu \mathrm{m}^{3}$ at a fixed $\mu=1.0 \mu \mathrm{m}^{3}$ (Table 1) were used for the prediction. Again, the survival probability shifted leftwards when the mean value $\mu$ increased from 0.5 (solid line) to 1.0 (dashed line) and 1.5 (dotted line) $\mu \mathrm{m}^{3}$ (Figure 5(c)), indicating that high $\mu$ value accelerated the decay of $\overline{P_{d V}}$ or $\overline{P_{a V}}$. No significant difference, however, was found when the standard deviation $\sigma$ increased from 0.1 (solid line) to 0.5 (dashed line) and 1.0 (dotted line) $\mu \mathrm{m}^{3}$ (Figure 5(d)), implying the insensitivity of effect of $\sigma$ on $\overline{P_{d V}}$ or $\overline{P_{a V}}$. Taken together, these predictions defined the time dependence of survival probability on varied volume distributions.

\subsection{Impact of time-dependent volume distribution}

It is evident that mitochondrial morphology depends on the net rate of its fission and fusion. At an equilibrium state, the fission rate is comparable to the fusion rate, which retains the same volume distribution. By contrast, the large-sized mitochondria have the low fraction in the pool of volume when the fusion rate reduces or vice verse when the fission rate lowers $[7,19]$. This was further confirmed in the current work where low fraction of large mitochondrial volume was observed in an intermitotic Hela cell with low mean value of $\beta\left(\bar{\beta}=0.97 \mu \mathrm{m}^{-3}\right)$ (cf. Figure 3(b)), as compared to that in a mitotic Hela cell $\left(\bar{\beta}=1.41 \mu \mathrm{m}^{-3}\right)$. It was also found from Figure 3(b) that both $\alpha$ and $\beta$ exhibited an ascending phase followed by a descending phase. Thus, it is important to quantify the impact of time-dependent Gamma distribution of mitochondrial volume on survival probability. Here two sets of formulation were proposed to describe the time dependence of $\alpha$ and $\beta$.

$$
\alpha(t)=\alpha_{0} \exp \left(-a_{1} t\right)
$$

and

$$
\beta(t)=\beta_{0} \exp \left(-a_{2} t\right) \quad \text { (for descending phase); }
$$

and

$$
\alpha(t)=\alpha_{0}+\Delta \alpha\left(1-\exp \left(-b_{1} t\right)\right),
$$

$\beta(t)=\beta_{0}+\Delta \beta\left(1-\exp \left(-b_{2} t\right)\right)$ (for ascending phase). (15b)

Here $\alpha_{0}$ and $\beta_{0}$ are the values at $t=0, \alpha_{0}+\Delta \alpha$ and $\beta_{0}+\Delta \beta$ are the values at $t \rightarrow+\infty$, and $a_{1}, a_{2}, b_{1}$, and $b_{2}$ are the characteristic time constants $\left(\right.$ in s$^{-1}$ ). Similar analytical solutions for
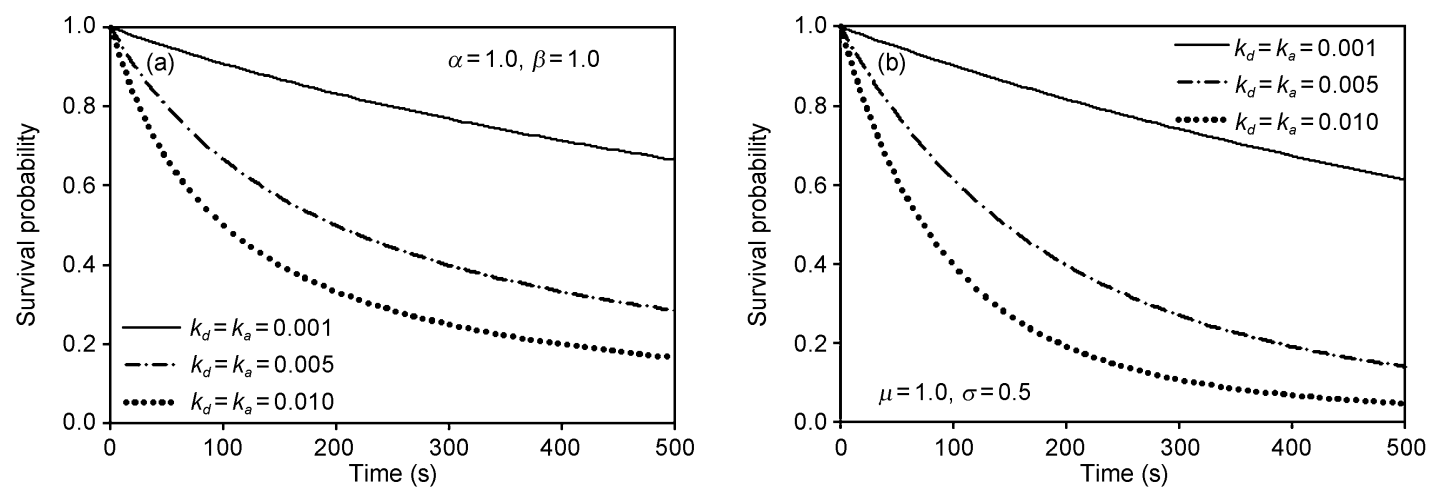

Figure 4 Time courses of mean survival probabilities $\overline{P_{d V}}$ and $\overline{P_{a V}}$, calculated using eqs. (11a) and (11b) for the Gamma-distributed volume at $\alpha=1.0$ and $\beta=1.0 \mu \mathrm{m}^{-3}$ (a) or using eqs. (13a) and (13b) for the Gaussian-distributed volume at $\mu=1.0 \mu \mathrm{m}^{3}, \sigma=0.5 \mu \mathrm{m}^{3}$, where $\overline{P_{d V}}$ or $\overline{P_{a V}}$ was re-normalized to 1.0 at $t=0$ (b). Here the fission and fusion rates $k_{d}$ and $k_{a}$ were set to be 0.001 (solid lines), 0.005 (dashed lines), and 0.010 (dotted lines) $\mathrm{s}^{-1} \mu \mathrm{m}^{-3}$, respectively. 

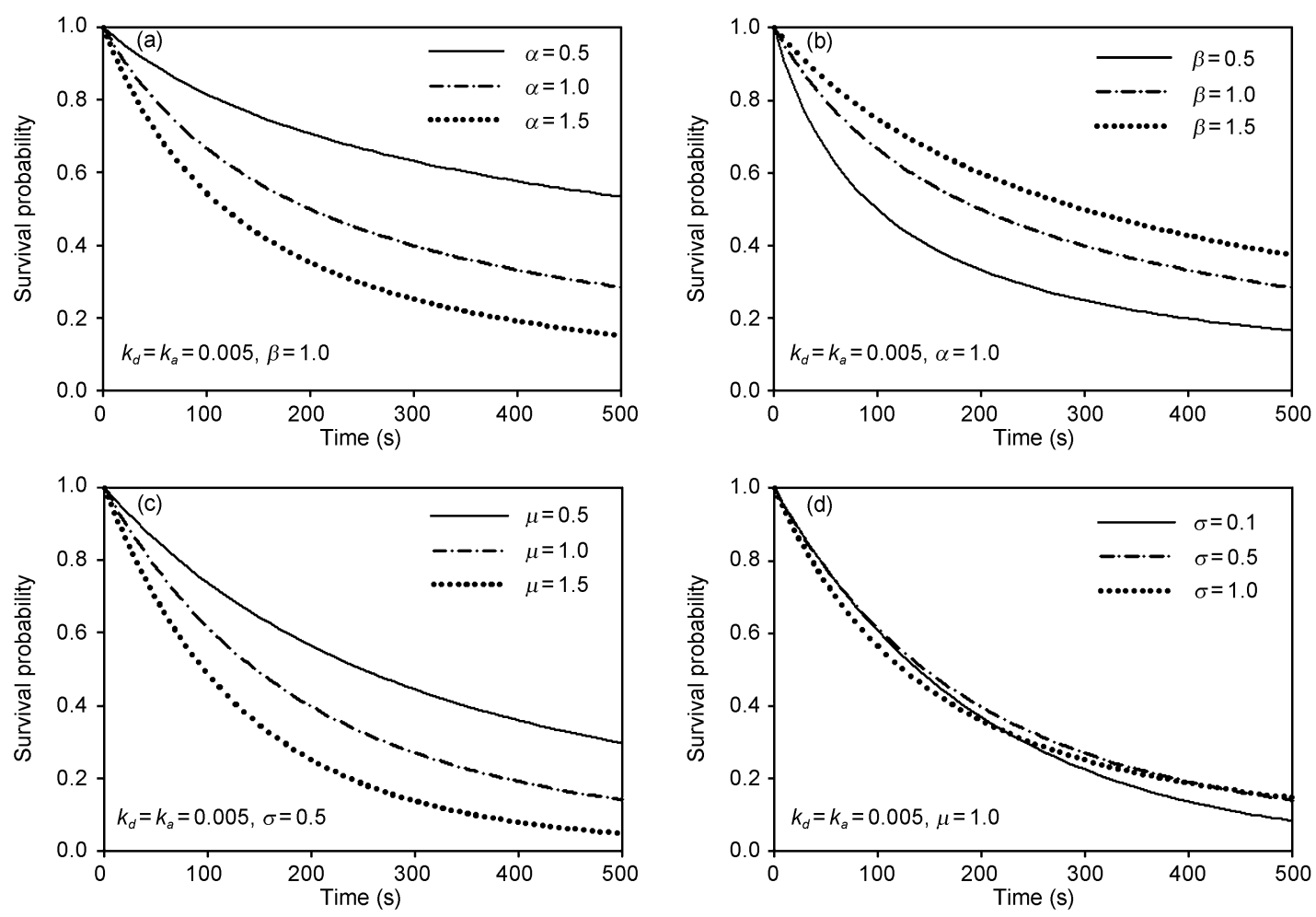

Figure 5 Time courses of mean survival probabilities $\overline{P_{d V}}$ and $\overline{P_{a V}}$, calculated using eqs. (11a) and (11b) for the Gamma-distributed volume when $\alpha$ was varied by 0.5 (solid line), 1.0 (dashed line), and 1.5 (dotted line) at a fixed $\beta=1.0 \mu \mathrm{m}^{-3}$ (a) or when $\beta$ was varied by 0.5 (solid line), 1.0 (dashed line), and 1.5 (dotted line) $\mu \mathrm{m}^{-3}$ at a fixed $\alpha=1.0$ (b) or using eqs. (13a) and (13b) for the Gaussian-distributed volume when $\mu$ was varied by 0.5 (solid line), 1.0 (dashed line), and 1.5 (dotted line) $\mu \mathrm{m}^{3}$ at a fixed $\sigma=0.5 \mu \mathrm{m}^{3}$ (c) or when $\sigma$ was varied by 0.1 (solid line), 0.5 (dashed line), and $1.0 \mu \mathrm{m}^{3}$ (dotted line) at a fixed $\mu=1.0 \mu \mathrm{m}^{3}$ (d). $\overline{P_{d V}}$ or $\overline{P_{a V}}$ was re-normalized to 1.0 at $t=0$ for Gaussian distribution ((c), (d)). Here the fission and fusion rates were set to be $k_{d}=$ $k_{a}=0.005 \mathrm{~s}^{-1} \mu \mathrm{m}^{-3}$.

mean survival probability $\overline{P_{d V}}$ or $\overline{P_{a V}}$ were obtained by substituting eqs. (14a) and (14b) or (15a) and (15b) into eqs. (6a) and (6b):

$$
\overline{P_{d V}}=\left(\frac{\beta(t)}{k_{d} t+\beta(t)}\right)^{\alpha(t)}
$$

or

$$
\overline{P_{a V}}=\left(\frac{\beta(t)}{k_{a} t+\beta(t)}\right)^{\alpha(t)}
$$

For the simplification of the predictions, the parameters were set to be $\alpha_{0}=\Delta \alpha=1.0$ and $\beta_{0}=\Delta \beta=1.0 \mu \mathrm{m}^{-3}$ and $a_{1}$ $=a_{2}=b_{1}=b_{2}=0.005 \mathrm{~s}^{-1}$. And the rate dependence of $\overline{P_{d V}}$ or $\overline{P_{a V}}$ was tested at $k_{d}=k_{a}=0.001$ (blue lines), 0.005 (red lines), and 0.01 (black lines) $\mathrm{s}^{-1} \mu \mathrm{m}^{-3}$ (Figures 6(a)-(d)). Four cases were involved in the following predictions: $\alpha(t)$ was defined by eq. (14a) (Figure 6(a)) or (15a) (Figure $6(\mathrm{~b})$ ) when $\beta(t) \equiv \beta_{0}$ and $\beta(t)$ was defined by eq. (14b) (Figure 6(c)) or (15b) (Figure 6(d)) when $\alpha(t) \equiv \alpha_{0}$ (solid lines), and then were compared with those at $\alpha(t) \equiv \alpha_{0}$ and $\beta(t) \equiv$ $\beta_{0}$ (dashed lines) (Figure 6). Again note that both $\overline{P_{d V}}$ and $\overline{P_{a V}}$ have the same forms when $k_{d}=k_{a}$ (eqs. (16a) and (16b)). The predictions indicated that $\overline{P_{d V}}$ or $\overline{P_{a V}}$ no longer decreased monotonically with time but exhibited a biphasic transition when it decreased at $t<100 \mathrm{~s}$ followed by an increase for descending phase of $\alpha(t)$ (eq. (14a)) (solid lines), while $\overline{P_{d V}}$ or $\overline{P_{a V}}$ retained a monotonic decrease at $\alpha(t) \equiv \alpha_{0}$ and $\beta(t) \equiv \beta_{0}$ (dash lines) (Figure 6(a)). This resulted in a significantly different pattern in $\overline{P_{d V}}$ or $\overline{P_{a V}}$ where it turned to be unity when $\alpha(t)$ approached zero at $t \rightarrow+\infty$. For a Gamma distribution (eq. (7)), the distribution of mitochondrial volume tended to be compressed towards the zero-volume regime and $\overline{P_{d V}}$ or $\overline{P_{a V}}$ approached unity at $\alpha \rightarrow 0$. These predictions suggested that the very small-sized mitochondria were not favorable for their fission or fusion activities. By contrast, $\overline{P_{d V}}$ or $\overline{P_{a V}}$ retained a monotonic decrease for ascending phase of $\alpha(t)$ (eq. (15a)) (solid lines) and shifted leftwards as compared to 

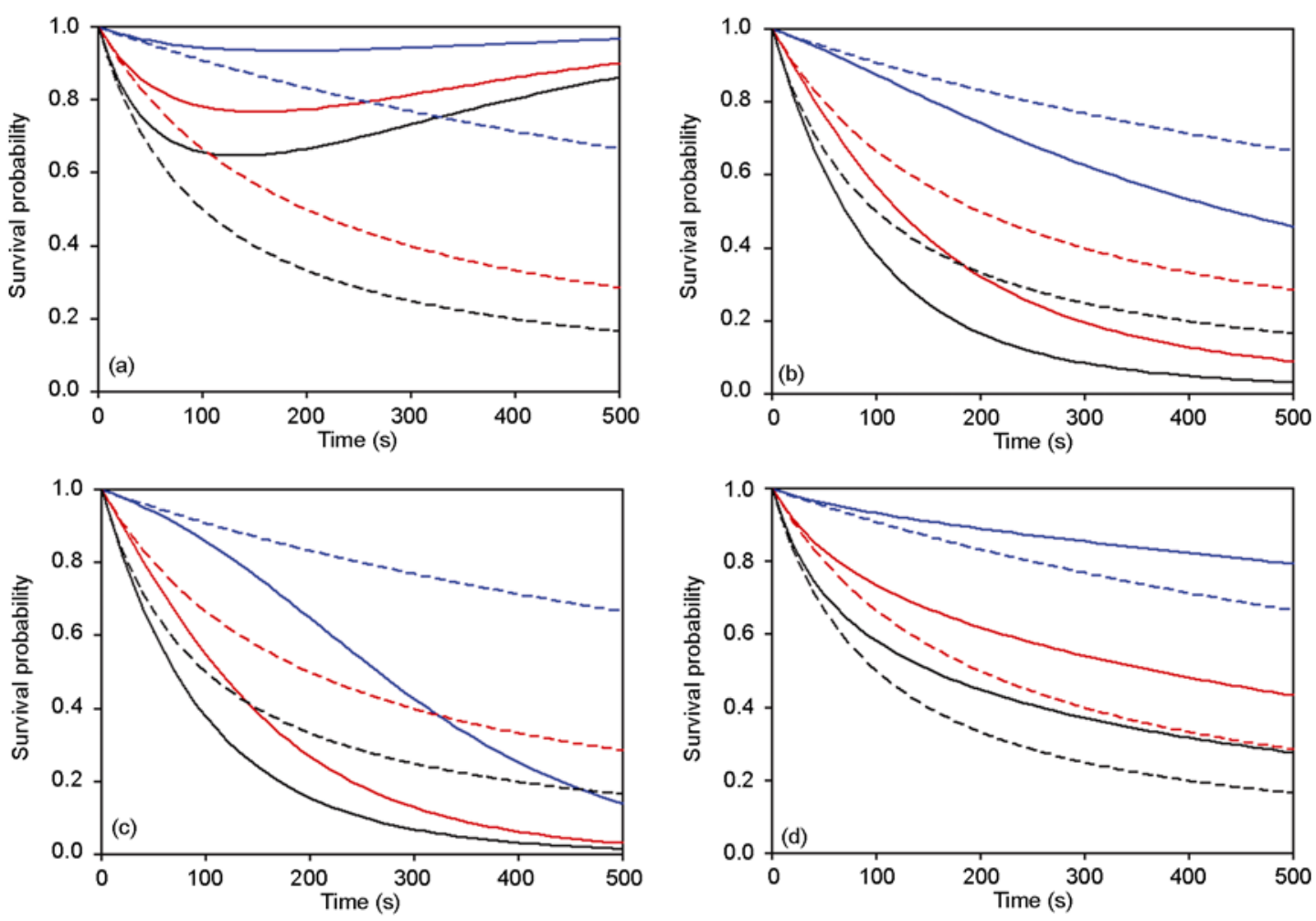

Figure 6 Comparison of time courses of mean survival probabilities, $\overline{P_{d V}}$ and $\overline{P_{a V}}$, between the time-dependent parameter set of $\alpha(t)$ and $\beta(t)$ and the constant value set of $\alpha_{0}$ and $\beta_{0}$. Plotted are the probabilities when $\alpha(t)$ was defined by eq. (14a) (a) or eq. (15a) (b) at a fixed $\beta(t) \equiv \beta_{0}=1.0 \mu \mathrm{m}^{-3}$ or when $\beta(t)$ was defined by eq. (14b) (c) or eq. (15b) (d) at a fixed $\alpha(t) \equiv \alpha_{0}=1.0$ (solid lines). Also plotted are the probabilities at the constant values of $\alpha(t) \equiv \alpha_{0}$ $=1.0$ and $\beta(t) \equiv \beta_{0}=1.0 \mu \mathrm{m}^{-3}$ (dashed lines). Here the parameters were set to be $\alpha_{0}=\Delta \alpha=1.0, \beta_{0}=\Delta \beta=1.0 \mu \mathrm{m}^{-3}$, and $a_{1}=a_{2}=b_{1}=b_{2}=0.005 \mathrm{~s}^{-1}$. The fission and fusion rates were set to be $k_{d}=k_{a}=0.001$ (blue lines), 0.005 (red lines), and 0.01 (black lines) $\mathrm{s}^{-1} \mu \mathrm{m}^{-3}$.

those at $\alpha(t) \equiv \alpha_{0}$ and $\beta(t) \equiv \beta_{0}$ (dashed lines) (Figure 6(b)), indicating that $\overline{P_{d V}}$ or $\overline{P_{a V}}$ decreased quickly and mitochondrial fission or fusion was active. No biphasic transition of $\overline{P_{d V}}$ or $\overline{P_{a V}}$ was found for either descending or ascending phase of $\beta(t)$ at $\alpha(t) \equiv \alpha_{0}$ (Figures $6(\mathrm{c})$ and (d)). Again, lower (Figure 6(c)) or higher (Figure 6(d)) $\overline{P_{d V}}$ or $\overline{P_{a V}}$ was presented for descending (eq. (14b)) or ascending (eq. (15b)) $\beta(t)$ (solid lines) than those at $\alpha(t) \equiv \alpha_{0}$ and $\beta(t) \equiv \beta_{0}$ (dashed lines), suggesting the higher or lower fission and fusion activities for time-dependent $\beta(t)$. Taken together, these predictions indicated that the varied Gamma distribution of mitochondrial volume was strongly correlated with their fission and fusion activities.

Finally, we developed a theoretical model to describe the dynamics of volume evolution for mitochondrial fission and fusion. The survival probability predicted from the model was found to be inversely correlated with the fission or fusion rate $\left(k_{d}\right.$ or $\left.k_{a}\right)$, which is applicable in quantifying the fission or fusion activities for mitochondrial physiology. This model could also be used to estimate the fission and fusion rates from the measurements of time course of survival probability and mitochondrial volume distribution in the future works.

The authors would like to thank XIAO WeiMing and JIANG ChunSun of the Institute of Zoology, Chinese Academy of Sciences, for assistance with experimental preparations. They would also express heartfelt thanks to the editors and referees of SCIENCE CHINA Physics, Mechanics of Astronomy for reviewing the manuscript. This work was supported by the National Natural Science Foundation of China (Grant Nos. 30730032 and 10332060), the National Key Basic Research Foundation of China (Grant No. 2006CB910303), the National High Technology Research and Development Program of China (Grant No. 2007AA02Z306), and the Knowledge Innovation Program of the Chinese Academy of Sciences (Grant No. KJCX2-YW-LO8).

1 Yaffe M P. The Machinery of Mitochondrial Inheritance and Behavior. Science, 1999, 283: 1493-1497

2 Scheffler I E. Mitochondria make a come back. Adv Drug Deliv Rev, 2001, 49: 3-26

3 Collins $\mathrm{T}$ J, Berridge $\mathrm{M} \mathrm{J}$, Lipp $\mathrm{P}$, et al. Mitochondria are morphologically and functionally heterogeneous within cells. EMBO J, 2002, 21: 1616-1627

4 de Giorgi F, Lartigue L, Ichas F. Electrical coupling and plasticity of the mitochondrial network. Cell Calcium, 2000, 28: 365-370

5 Rizzuto R, Pinton P, Carrington W, et al. Close contacts with the endoplasmic reticulum as determinants of mitochondrial $\mathrm{Ca}^{2+}$ responses. Science, 1998, 280: 1763-1766

6 Meeusen S, McCaffery M, Nunnari J. Mitochondrial fusion intermediates revealed in vitro. Science, 2004, 305: 1747-1752 
7 Taguchi N, Ishihara M, Jofuku A, et al. Mitotic phosphorylation of dynamin-related GTPase Drp1 participates in mitochondrial fission. J Biol Chem, 2007, 282: 11521-11529

8 Chen H, Chomyn A, Chan D C. Disruption of fusion results in mitochondrial heterogeneity and dysfunction. J Biol Chem, 2005, 280: 26185-26192

9 Miller K E, Sheetz M P. Axonal mitochondrial transport and potential are correlated. J Cell Sci, 2004, 117: 2791-2804

10 Miller K E, Sheetz M P. Direct evidence for coherent low velocity axonal transport of mitochondria. J Cell Biol, 2006, 117: 373-381

11 Knowles M K, Guenza M G, Capaldi R A, et al. Cytoskeletal-assisted dynamics of the mitochondrial reticulum in living cells. Proc Natl Acad Sci USA, 2002, 99: 14772-14777

12 Tang X, Liu J, Wu B. Fractal property of the cytoskeleton in the living cells. Chin Phys Lett, 1994, 11: 522-525

13 Li D, Xiong J, Qu A, et al. Three-dimensional tracking of single secretory granules in live PC12 cells. Biophys J, 2004, 87: 1991-2001

14 Fehrenbacher K L, Yang H, Gay A C, et al. Live cell imaging of mitochondrial movement along actin cables in budding yeast. Curr
Biol, 2004, 14: 1996-2004

15 Nunnari J, Marshall W F, Straight A, et al. Mitochondrial transmission during mating in Saccharomyces cerevisiae is determined by mitochondrial fusion and fission and the intramitochondrial segregation of mitochondrial DNA. Mol Biol Cell, 1997, 8: 1233-1242

16 Gorsich S W, Shaw J M. Importance of mitochondrial dynamics during meiosis and sporulation. Mol Biol Cell, 2004, 15: 4369-4381

17 Berman S B, Pineda F J, Hardwick J M. Mitochondrial fission and fusion dynamics: The long and short of it. Cell Death Differ, 2008, 15: 1147-1152

18 Huang P Y, Hellums J D. Aggregation and disaggregation kinetics of human blood platelets: Part 1. Development and validation of a population balance method. Biophys J, 1993, 65: 334-343

19 Ishihara N, Jofuku A, Eura Y, et al. Regulation of mitochondrial morphology by membrane potential, and DRP1-dependent division and FZO1-dependent fusion reaction in mammalian cells. Biochem Biophys Res Commun, 2003, 301: 891-898

20 Wang S Q, Jiang C S, Zhang Y, et al. Membrane deformability and membrane tension of single isolated mitochondria. Cell Mol Bioeng, 2008, 1: 67-74 